\title{
From Medicinal Chemistry to Human Health: Current Approaches to Drug Discovery for Cancer and Neglected Tropical Diseases
}

\author{
LEONARDO G. FERREIRA, GLAUCIUS OLIVA and ADRIANO D. ANDRICOPULO
}

\begin{abstract}
Laboratório de Química Medicinal e Computacional, Centro de Pesquisa e Inovação em Biodiversidade e Fármacos, Instituto de Física de São Carlos, Universidade de São Paulo, Av. João Dagnone, 1100, 13563-120 São Carlos, SP, Brazil
\end{abstract}

Manuscript received on July 3, 2017; accepted on August 9, 2017

\begin{abstract}
Scientific and technological breakthroughs have compelled the current players in drug discovery to increasingly incorporate knowledge-based approaches. This evolving paradigm, which has its roots attached to the recent advances in medicinal chemistry, molecular and structural biology, has unprecedentedly demanded the development of up-to-date computational approaches, such as bio- and chemo-informatics. These tools have been pivotal to catalyzing the ever-increasing amount of data generated by the molecular sciences, and to converting the data into insightful guidelines for use in the research pipeline. As a result, ligand- and structure-based drug design have emerged as key pathways to address the pharmaceutical industry's striking demands for innovation. These approaches depend on a keen integration of experimental and molecular modeling methods to surmount the main challenges faced by drug candidates - in vivo efficacy, pharmacodynamics, metabolism, pharmacokinetics and safety. To that end, the Laboratório de Química Medicinal e Computacional (LQMC) of the Universidade de São Paulo has developed forefront research on highly prevalent and life-threatening neglected tropical diseases and cancer. By taking part in global initiatives for pharmaceutical innovation, the laboratory has contributed to the advance of these critical therapeutic areas through the use of cutting-edge strategies in medicinal chemistry.
\end{abstract}

Key words: medicinal chemistry, ligand-based drug design, structure-based drug design, cancer, neglected tropical diseases.

\section{INTRODUCTION}

Modern drug research and development (R\&D) has experienced remarkable changes as technological and scientific developments in the past years have dramatically changed the pharmaceutical innovation process. Well-established strategies,

Correspondence to: Adriano Defini Andricopulo

E-mail: aandrico@ifsc.usp.br

* Contribution to the centenary of the Brazilian Academy of Sciences. such as high-throughput screening (HTS), have progressively been applied in association with novel techniques founded on genomics, molecular and structural biology and molecular modeling (Taboureau et al. 2012). Altogether, these cuttingedge fields have provided outstanding advances for our comprehension of the fundamental cellular and molecular mechanisms of diseases in addition to enabling important progress in the technological arsenal used in drug discovery. Combined with 
novel methods in organic synthesis, such as combinatorial chemistry, these forefront approaches have built a novel paradigm in the research-based pharmaceutical industry (Dopazo 2014).

The incorporation of molecular and computational strategies to drug development along with organic synthesis approaches has led to a sharp increase in the availability of biological, structural and chemical data. Additionally, this evolving scenario has caused a remarkable rise in the complexity of the $R \& D$ process (Juliano 2013). As a result, current drug discovery has become strongly dependent on the establishment of closely connected experimental and computational research platforms. The implementation of this type of strategy has become pivotal to extract knowledge from the generated data and predict key events related to drug action and safety. The importance of these knowledge-based approaches in medicinal chemistry has been corroborated by the growing number of publications communicating the use of these strategies in the identification of promising drug candidates, many of them going through clinical trials and being approved for clinical use (Eder et al. 2014).

These modern R\&D strategies usually combine structure- and ligand-based drug design methods (SBDD and LBDD, respectively) and many different computational and experimental techniques. Widely used SBDD technologies, $\mathrm{X}$-ray crystallography, molecular docking and structure-based virtual screening have provided important insights into ligand-receptor molecular recognition. On the other side, LBDD techniques, such as pharmacophore modeling, quantitative structure-activity relationships (QSAR), and ligand-based virtual screening, have been broadly used to probe small-molecule virtual databases and identify correlations between biological activity and chemical structure (Trossini et al. 2009, Castilho et al. 2009). Additionally, as a core approach in modern pharmaceutical $\mathrm{R} \& \mathrm{D}$, quantitative structure-property relationships (QSPR) have been pivotal for estimating events associated with drug absorption, distribution, metabolism, excretion and toxicity (ADMET) (Berhanu et al. 2012).

\section{STRUCTURE- AND LIGAND-BASED DRUG DESIGN}

SBDD is the use of $3 \mathrm{D}$ structures of molecular targets to enhance ligand-receptor complementarity and improve parameters such as potency, affinity and selectivity. The 3D structures used in SBDD are mainly obtained by biophysical techniques such as X-ray crystallography and nuclear magnetic resonance (Surade and Blundell 2012). The use of SBDD approaches, specifically in the early stages of the R\&D process, has allowed for the determination of 3D structures of many pharmacological targets and a precise delineation of their binding site characteristics, such as shape and electrostatic features. By unraveling these aspects, SBDD strategies enable the design of ligands with appropriate structural and physicochemical properties for an optimum interaction with the molecular target. The outlining and identification of putative ligands can be assisted by molecular modeling methods such as molecular docking and structure-based virtual screening (Ferreira et al. 2015). Promising ligands, called hits, identified by these approaches can be subsequently tested for their potency and affinity against the molecular target. Once active hits are identified, they can be characterized regarding their binding mode by biophysical techniques, providing key information about the molecular aspects that drive the ligandreceptor interaction. Furthermore, characterizing the ligand-receptor binding mode enables the formulation of relationships between biological activity and structural data. Depending on these findings, medicinal chemistry approaches, including structure-activity relationship (SAR) studies, are performed to optimize the ligand 
properties (mainly those related to the affinity for the molecular target).

LBDD approaches do not require knowledge of the receptor $3 \mathrm{D}$ structure. Instead, these studies demand information about the structure, activity, and other properties of compounds that are known to be active toward the investigated disease (Berhanu et al. 2012). These data are used as inputs for the construction of QSAR and QSPR models that correlate the ligand properties, called molecular descriptors or independent variables, with pharmacodynamics or pharmacokinetics parameters, which are the dependent variables (Stanton 2012). These chemometric models are able to identify the ligand properties that more critically affect the dependent variable. Moreover, they are useful for conceiving novel compounds and predicting their dependent variables before synthesis and experimental evaluation. Also broadly applied in LBDD, pharmacophore models are used to identify the sites among a series of molecules that are most likely to engage in favorable molecular interactions with the molecular target (Baig et al. 2016).

Both types of strategies, SBDD and LBDD, aim to identify novel hits and optimize them into lead compounds and ultimately into drug candidates. To that end, the identified compounds undergo a number of experimental evaluations that are relevant for the disease under investigation. The purpose of these investigations is to simultaneously optimize pharmacodynamics, pharmacokinetics and safety parameters. This is critical since in vivo drug efficacy depends on a sharp balance between potency toward the molecular target and suitable ADMET properties (Kirchmair et al. 2015). Generally, these parameters are assessed by different types of in vitro techniques that can provide key information for use in the molecular optimization process when integrated with computational studies.
EXPERIMENTAL EVALUATION METHODS IN DRUG DISCOVERY

In vitro profiling for assessing biological activity is generally divided into target-based and phenotypic approaches (Swinney 2013). Target-based methods are broadly used and are aimed at measuring the effect of the ligand toward the activity of the molecular target. Generally, they consist of binding, competition and enzymatic bioassays performed on the purified receptor and enzyme, whereby several useful parameters can be obtained. Among these parameters, we can highlight the concentration of inhibitor required to inhibit the activity of an enzyme by $50 \%\left(\mathrm{IC}_{50}\right)$ and the receptor-inhibitor dissociation constant $\left(K_{\mathrm{i}}\right)$ (Copeland 2000). In general, the molecular target samples used in these assays are obtained via recombinant DNA technologies or through purification from the biological tissues in which they are expressed (Overton 2014).

On the other hand, phenotypic approaches assess the ability of test compounds to produce biological effects in whole cells (Swinney 2013). A common procedure is the evaluation of cell viability upon treatment with test compounds. These viability tests are broadly used in pharmaceutical R\&D and have the advantages of determining the effect of compounds in the complex cellular environment and assessing cell uptake issues. Additionally, the use of normal human cells allows the evaluation of compound-induced cytotoxic effects. However, optimization of phenotypic hits in the absence of any information about the receptor $3 \mathrm{D}$ structure may be more challenging compared with targetbased approaches (Swinney and Anthony 2011).

In addition to the evaluation of pharmacodynamics aspects, such as biological activity (e.g., potency, affinity, selectivity), pharmacokinetics assessment is critical in modern drug R\&D. Poor pharmacokinetics is one of the leading causes for failure of drug candidates; thus, 
pharmaceutical companies have invested in several novel strategies aimed at the early determination and prediction of absorption, distribution, metabolism and excretion (ADME) properties (Di 2015). Pharmacokinetics is strongly dependent on the physicochemical parameters of the compounds, which determine the permeability of the drugs through several biological barriers imposed by the human body and, consequently, their ability to reach the molecular target site. It is also critical the amenability of the drug to undergo chemical transformations via metabolism enzymes that are mainly located in the liver. This process is required for converting highly lipophilic molecules to water-soluble metabolites that can be more easily eliminated from the body (Testa et al. 2012).

Considering these pharmacokinetics aspects, developing effective orally administered drugs is the main goal of pharmaceutical R\&D. This requires the absorption of the drug candidate through the gastrointestinal epithelium and subsequent access to the bloodstream (Bergström et al. 2014). Failure to cross this biological barrier causes suboptimal bioavailability, which can lead to reduced or a lack of efficacy. Usually, in vitro techniques are used to evaluate human intestinal absorption (HIA) during the early stages of drug discovery. The assay based on Caco-2 cells, which are obtained from colon adenocarcinoma tumors, is the most used technique in the pharmaceutical industry to estimate HIA (Volpe 2011). The reliability of the Caco-2 model to evaluate drug permeability lies in the ability of these cells to acquire characteristics of the intestinal epithelium. This technique is used to evaluate both passive transport (through the cells or the cellular junctions) and active transport mediated by carriers such as P-glycoprotein (P-gp). Another frequently used technique, which is restricted to evaluating passive transport, is based on the Parallel Artificial Membrane Permeability Assay (PAMPA) (Volpe 2011). The PAMPA assay is more straightforward to execute and can yield results comparable to those obtained with the Caco-2 assay; therefore, it is a useful alternative to quickly deliver HIA estimates when large compound collections need to be screened.

Another critical matter in pharmaceutical $R \& D$ is the evaluation of drug metabolism. To that end, several techniques are currently available to measure the metabolic stability of drug candidates. These tests are additionally useful for estimating the potential for the emergence of drug-drug interactions (DDIs) due to the modulation of metabolism-associated enzymes (Galetin et al. 2010). Usually, the pharmaceutical industry conducts in vitro metabolic stability studies along with computational investigations (Honório et al. 2013). The evaluation of metabolic liabilities in the early phases of the discovery process is pivotal to exclude compounds that have unsuitable properties and design new ones that have enhanced profiles.

Among the available methods to conduct metabolic stability studies, the technique based on liver microsomes has become a widely used in vitro model (Asha and Vidyavathi 2010). These organelles contain a number of enzymes with the most important ones belonging to the cytochrome P450 (CYP) superfamily, which is involved in phase I metabolic reactions, such as oxidation, reduction and hydrolysis (Testa et al. 2012). Cytochrome P450 enzymes are responsible for the metabolism of $90 \%$ of commercially available drugs. The most abundant isoform in the human liver, CYP3A4, is implicated in the metabolism of $50 \%$ of all marketed drugs and in a number of clinically relevant DDIs (Baranczewski et al. 2006). The metabolic stability toward liver microsomal enzymes is determined based on the loss of the parent compound as measured at different time points. Since the output of these experiments is expressed as in vitro halflife $\left(\mathrm{T}_{1 / 2}\right)$ and intrinsic clearance $\left(\mathrm{CL}_{\text {int }}\right)$, metabolic stability studies can provide an estimate of important secondary pharmacokinetic parameters, such as hepatic clearance and bioavailability 
(Baranczewski et al. 2006). If a molecule is quickly metabolized, its in vivo bioavailability is likely to be low and, therefore, molecular optimization efforts can be performed to improve its pharmacokinetics profile.

\section{DRUG DISCOVERY AT THE LABORATORY OF MEDICINAL AND COMPUTATIONAL CHEMISTRY}

SBDD and LBDD have been applied as forefront drug discovery approaches both in academic and industrial environments. In this context, these strategies have been employed over the past 15 years in a number of investigations at the Laboratório de Química Medicinal e Computacional (LQMC) in the Instituto de Física de São Carlos (IFSC) at the Universidade de São Paulo (USP). Life-threatening conditions, such as cancer, and neglected tropical diseases (NTDs) (e.g., schistosomiasis, leishmaniasis and Chagas disease) have been investigated via the integrated use of computational and experimental strategies in SBDD and LBDD (Postigo et al. 2011). These studies have combined several types of target-based and phenotypic assays and bio- and chemo-informatics approaches to investigate key aspects related to pharmacokinetics and pharmacodynamics (Moda and Andricopulo 2012). To this end, leading-edge techniques, such as Caco-2, PAMPA and metabolic stability methods, have been implemented in LQMC. In addition, the laboratory team has developed chemoinformatics tools, such as highly predictive QSPR models, to estimate ADME properties (Moda

\section{STRUCTURE-BASED DRUG DESIGN}

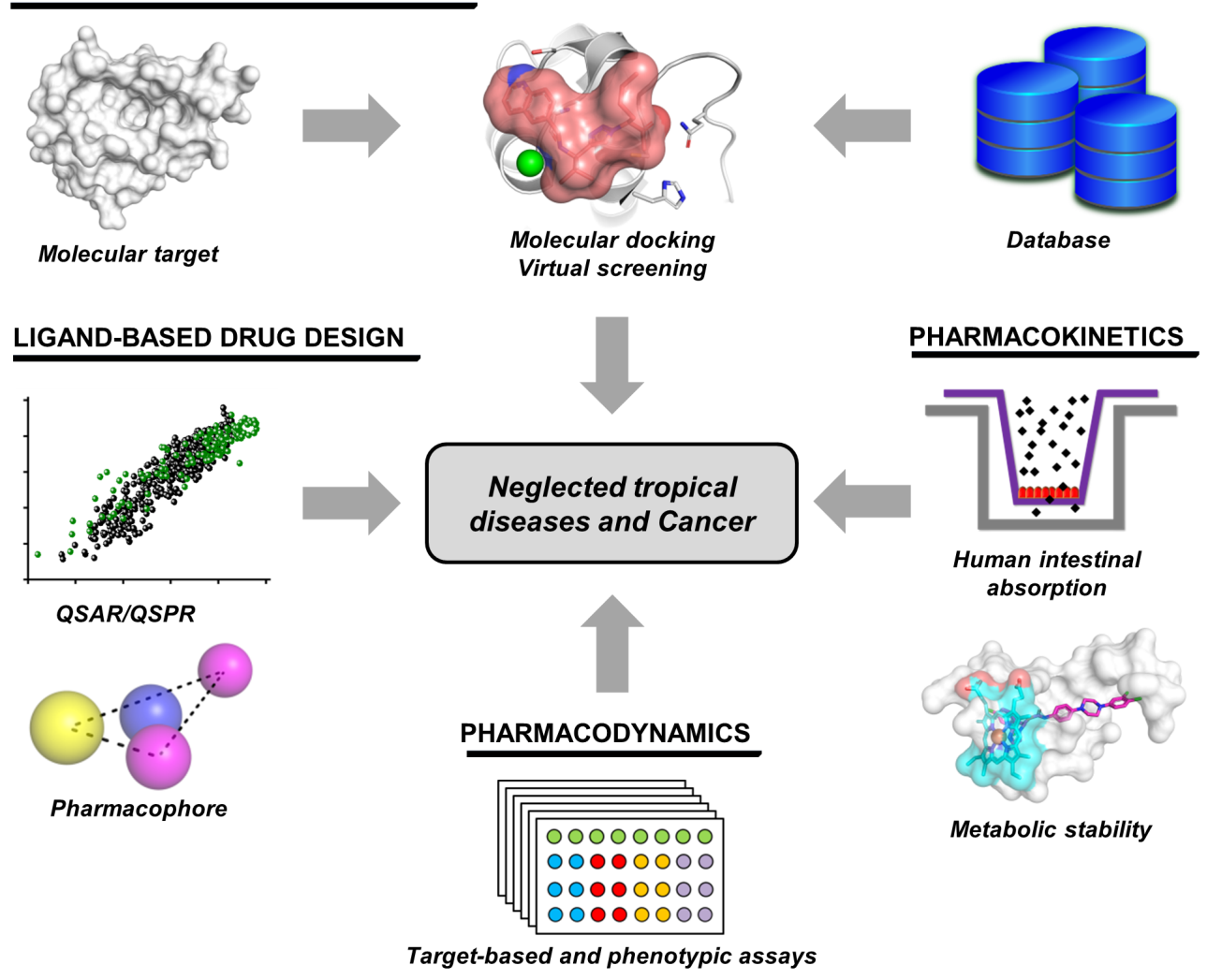

Figure 1 - Drug discovery strategies employed at the Laboratory of Medicinal and Computational Chemistry (LQMC). Ligand- and structure-based drug design methods are used alongside several computational and experimental techniques to evaluate pharmacodynamics and pharmacokinetics in cancer and tropical disease drug discovery. 
et al. 2008). In this context, the drug discovery approach applied at LQMC demonstrates the full integration of molecular modeling, LBDD, SBDD, and target-based and phenotypic strategies for the investigation of drug activity and ADME parameters (Fig. 1). In the next sections, selected studies exemplifying the research fields explored at LQMC will be highlighted.

\section{CANCER}

Cancer is the name given to a group of more than 100 diverse diseases that are characterized by abnormal reproduction of cells (Fouad and Aanei 2017). It is a highly prevalent condition and one of the leading causes of death worldwide. Nearly 15 million cases of cancer occur each year, leading to approximately 8.2 million deaths (Global Burden of Disease Cancer Collaboration et al. 2015). Despite the availability of a number of chemotherapeutic agents, the serious side effects associated with their use and drug resistance are major drawbacks in cancer therapy. To address these shortcomings, SBDD studies focused on several pharmacological targets have been explored in cancer R\&D.

Microtubules are among the most studied molecular targets in cancer drug discovery. They form the cell cytoskeleton and participate in key biological processes such as cell reproduction, migration and intracellular transport (Mukhtar et al. 2014). Microtubules consist of $\alpha$ and $\beta$ tubulin heterodimers whose process of assembly and disassembly is essential for their biological function. Any disturbance in these dynamics interferes with vital processes such as the formation of the mitotic spindle (Lecland and Lüders 2014). Impairing mitotic spindle formation hinders mitosis and consequently leads to apoptosis. Therefore, tubulin has been intensively explored as a molecular target in cancer drug discovery. The value of targeting tubulin has been demonstrated by the use of anticancer drugs such as paclitaxel, vinblastine and vincristine (Seligmann and Twelves 2013). Similar to other compounds targeting tubulin, these agents are broadly classified as microtubule-stabilizing drugs, such as paclitaxel, and microtubule-destabilizing molecules, such as vinblastine, vincristine and colchicine.

The use of these anticancer compounds is associated with several pharmacokinetics, toxicity and drug resistance issues. For example, colchicine, a natural tricyclic alkaloid, is highly toxic (Medani and Wall 2016). Vinca alkaloids, such as vincristine and vinblastine, and taxanes, such as paclitaxel, have complex structures and many chiral centers, rendering their synthesis exceptionally challenging (Howat et al. 2014). Podophyllotoxin (1), another highly potent natural product that acts as a microtubule-destabilizer, is also remarkably toxic (Kamal et al. 2015). Given this context, less toxic compounds that have low structural complexity are greatly needed as new microtubule modulating agents.

Therefore, we have developed a drug discovery strategy based on the structure of podophyllotoxin (Fig. 2). With the goal of identifying structurally non-complex molecules resembling the binding properties of podophyllotoxin, this approach led to the characterization of a series of acridinones as novel microtubule destabilizers (Magalhaes et al. 2016). Following this strategy, a series of azaanalogues of podophyllotoxin that contain only one chiral center was synthesized using a one-step multicomponent reaction. These compounds were designed to interact with the colchicine binding pocket at the tubulin $\alpha \beta$ interface and consequently prevent polymerization. The conception of the compounds was assisted by molecular docking studies that showed a high complementarity between the acridinone moiety of the inhibitors and the colchicine binding cavity at the $\alpha \beta$ interface.

After the molecular docking studies and synthesis, several target-based and phenotypic assays were performed. First, the anti-migratory 
<smiles></smiles>

Podophyllotoxin (1)

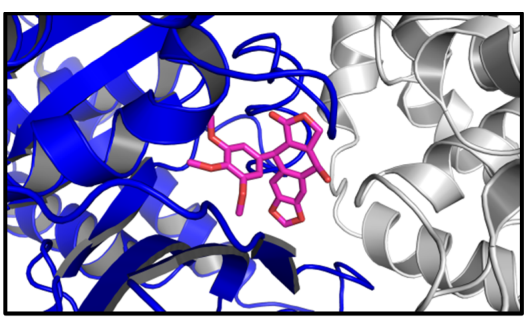

Tubulin in complex with compound 1

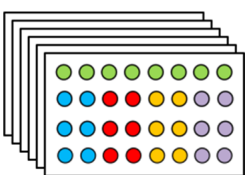

Cell viability

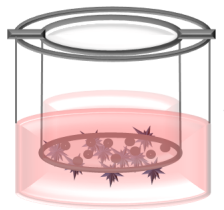

Migration assay

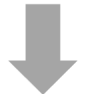

Molecular docking

Drug design

Synthesis of analogs

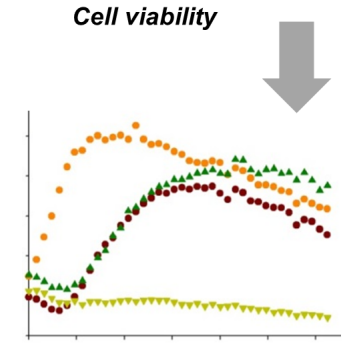

Tubulin polimerization

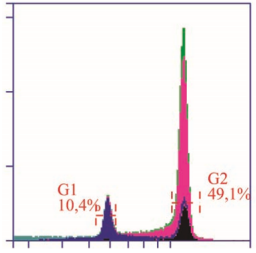

$1_{149,10 \%}$

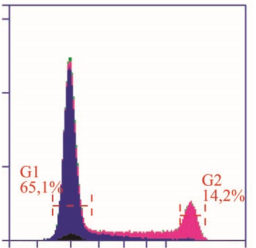

Cell cycle analyses
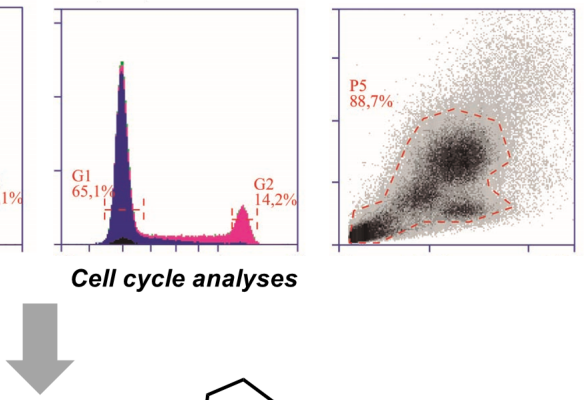

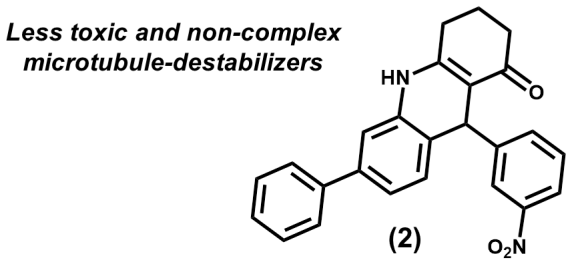<smiles>COc1cc(C2C3=C(CCCC3=O)Nc3cc(-c4ccccc4)ccc32)cc(OC)c1OC</smiles>

Figure 2 - Medicinal chemistry approaches used in the identification of novel microtubule destabilizing agents. By using podophyllotoxin as the reference compound, the integration of molecular modeling, organic synthesis and target-based and phenotypic methods led to discovery of a novel class of noncomplex tubulin polymerization inhibitors.

activity of the designed molecules was assessed since cell migration is dependent on microtubule formation. Some of the compounds were found to be potent cell migration inhibitors, as observed in Boyden chamber migration assays. Compounds 2 and 3 showed $\mathrm{IC}_{50}$ values of 0.29 and $0.33 \mu \mathrm{M}$, respectively, which are comparable with that of colchicine $\left(\mathrm{IC}_{50}=0.32 \mu \mathrm{M}\right)$. Next, these acridinones were evaluated for their cytotoxicity effects toward MDA-MB-231 cancer cell lines. $\mathrm{IC}_{50}$ values of 0.19 and $0.11 \mu \mathrm{M}$ were obtained for compounds 2 and $\mathbf{3}$, respectively. The activity of these compounds toward normal human fibroblasts was evaluated, and the results showed a high selectivity ( $\sim 30$-fold) for cancer cell lines, indicating a low potential for toxic effects.

In a next step, target-based studies were conducted to evaluate the ability of the designed molecules to inhibit tubulin assembly. As predicted by the molecular modeling studies, active 
acridinones were able to inhibit the assembly of purified tubulin. Compounds $\mathbf{2}$ and $\mathbf{3}$ displayed the most promising profiles, yielding $\mathrm{IC}_{50}$ values of 2.4 and $0.9 \mu \mathrm{M}$, respectively, which are comparable to those obtained for colchicine. Competition assays confirmed that the designed inhibitors produce their microtubule-destabilizing effects by interacting with the colchicine binding cavity. Furthermore, cell cycle analyses showed that the most active inhibitors act as mitotic arresters, which is consistent with their microtubule-destabilizing activity. In addition, these compounds could induce cell apoptosis on a level similar to that of colchicine ( $65 \%$ of apoptotic cells after $24 \mathrm{~h}$ ). In short, this medicinal chemistry strategy was able to identify novel, less toxic and non-complex microtubule destabilizers that were synthesized in a one-step reaction. The data gathered in this work render this series a promising starting point for further studies, which are in progress in our laboratory.

\section{NEGLECTED TROPICAL DISEASES (NTDs)}

The term NTDs is used to classify a group of 18 conditions predominantly caused by parasitic organisms that mainly affect low-income countries. Nearly one billion people suffer from these diseases, particularly in Africa, Latin America, and Asia (Ferreira and Andricopulo 2016). Additionally, as a result of increasing population mobility worldwide, developed nations have recently been affected by NTDs, which has contributed to the advance of global awareness of these conditions. This phenomenon has led to a consistent upgrading of research efforts toward NTDs, which are mainly supported by global publicprivate partnerships. Involving the pharmaceutical industry, philanthropic organizations and research institutions, these cooperative networks have promoted the transference of cutting-edge technologies to the NTDs arena and have been the forefront organizational models for drug discovery in this field (Starr et al. 2016). In this context, one of the leading organizations in this area, the Drugs for Neglected Diseases Initiative (DNDi), has established a drug discovery program called the Lead Optimization Latin America (LOLA) (https://www.dndi.org/2013/media-centre/newsviews-stories/news/first-early-stage-researchlatin-america/). The goal of this program is to foster early-stage research on NTDs focused on the specific needs of the region. LQMC plays a key role in this partnership as the center for medicinal chemistry, drug design and biological and biochemical screening for leishmaniasis and Chagas disease. As a central partner in this effort, LQMC has worked alongside DND $i$, the Institute of Chemistry at the University of Campinas (UNICAMP), and multinational pharmaceutical companies to develop improved therapies for these highly prevalent diseases in Latin America.

\section{CHAGAS DISEASE}

Chagas disease is an NTD caused by the kinetoplastid parasite Trypanosoma cruzi, which affects between 8 and 10 million people worldwide. The disease kills more than 10,000 people each year and threatens approximately 70 million with the risk of infection (Ferreira et al. 2016). Chagas disease is endemic in 21 countries in Latin America, where it is the main cause of heart failure. Recently, the disease has become a public health concern in wealthy nations, mainly in the USA, where nearly 240,000 cases have been registered (Manne-Goehler et al. 2016).

Chemotherapy of Chagas disease is restricted to two obsolete drugs: (i) benznidazole, a nitroimidazole discovered in the early 1970s and (ii) nifurtimox, a nitrofuran identified in the mid1960s. These drugs have several drawbacks, among which complete inefficacy in the chronic phase of the disease is the most relevant. In addition, serious adverse effects, such as skin reactions, 
gastrointestinal disorders, neuronal toxicity and fever, frequently result in a lack of commitment to treatment especially in adults (Coura and BorgesPereira 2011). Benznidazole is less toxic than nifurtimox; thus, it is used as the first-line drug. These shortcomings demonstrate the immediate demand for novel therapeutic agents for Chagas disease.

Current SBDD approaches to Chagas disease drug discovery have focused on various T. cruzi molecular targets, among which cruzain has been one of the most explored. This enzyme, the main cysteine protease from $T$. cruzi, is expressed during the entire life cycle of the parasite and is vital for its nutrition, reproduction, invasion of host cells and evasion from the immune system (Ferreira and Andricopulo 2017). The validation of cruzain as a pharmacological target relies on genetic and chemical validation experiments, which have demonstrated the essential role of the enzyme for parasite survival and infectivity (Doyle et al. 2011). In addition, preclinical proof-of-concept studies have confirmed that cruzain inhibitors are able to clear parasite burden in animal models of the disease (Doyle et al. 2007).

As part of our current research activities on the identification of novel cruzain inhibitors, a series of benzimidazole derivatives were designed and evaluated (Fig. 3). Benzimidazoles were previously identified as reversible cruzain inhibitors in an integrated HTS and virtual screening approach. This campaign resulted in the discovery of compound 4, which has an $\mathrm{IC}_{50}$ value of $800 \mathrm{nM}$ toward the enzyme (Ferreira et al. 2010). This compound was co-crystallized with the enzyme, and the X-ray structure (PDB 3KKU, $1.28 \AA$ ) revealed important aspects regarding the ligand-receptor molecular recognition. For example, the amide group of $\mathbf{4}$ was found to engage in a hydrogen bond network with the backbone of Asp161 and Gly66. The bromophenyl moiety was observed to interact with a key hydrophobic pocket of the active site, called the S2 subsite, and the benzimidazole group was found to build hydrogen bond interactions with water molecules.

Taking into account the crystallographic information and the high inhibitory activity of 4, medicinal chemistry efforts were undertaken, resulting in the synthesis of more than 40 derivatives that were evaluated against cruzain (Ferreira et al. 2014). The molecular optimization process was supported by molecular docking simulations, which were useful to evaluate the role of each ligand moiety for the ligand-receptor molecular recognition. The results of the enzymatic assays were used to generate important SAR data. For example, it was observed that switching the bromophenyl group with a bromonaphthyl and consequently increasing the hydrophobicity and steric bulk in this region results in an activity gain toward the enzyme $\left(\mathbf{5}, \mathrm{IC}_{50}=210 \mathrm{nM}\right)$. According to the molecular docking results, the bromonaphthyl moiety is able to fill the whole extension of the notably hydrophobic S2 subsite, an aspect that was recognized as the cause of the activity enhancement. Another relevant finding is the critical role played by the benzimidazole group, whose replacement with different cyclic systems results in a remarkable reduction in the biological activity. Out of the evaluated cruzain inhibitors, the lead compound $\mathbf{4}$ showed the most promising activity in phenotypic assays against $T$. cruzi $\left(\mathrm{IC}_{50}\right.$ $=1.63 \mu \mathrm{M})$.

Recently, these compounds have been evaluated for several pharmacokinetics parameters, such as HIA and metabolic stability. Thus far, the gathered results have indicated suitable ADME profiles. Furthermore, in vivo tolerability experiments disclosed no acute toxicity reactions in mice. In addition, QSAR models have been constructed to identify the key structural features that are critical to the biological activity of this series (Pauli et al. 2017). Altogether, these studies have resulted in the identification of novel drug-like compounds 


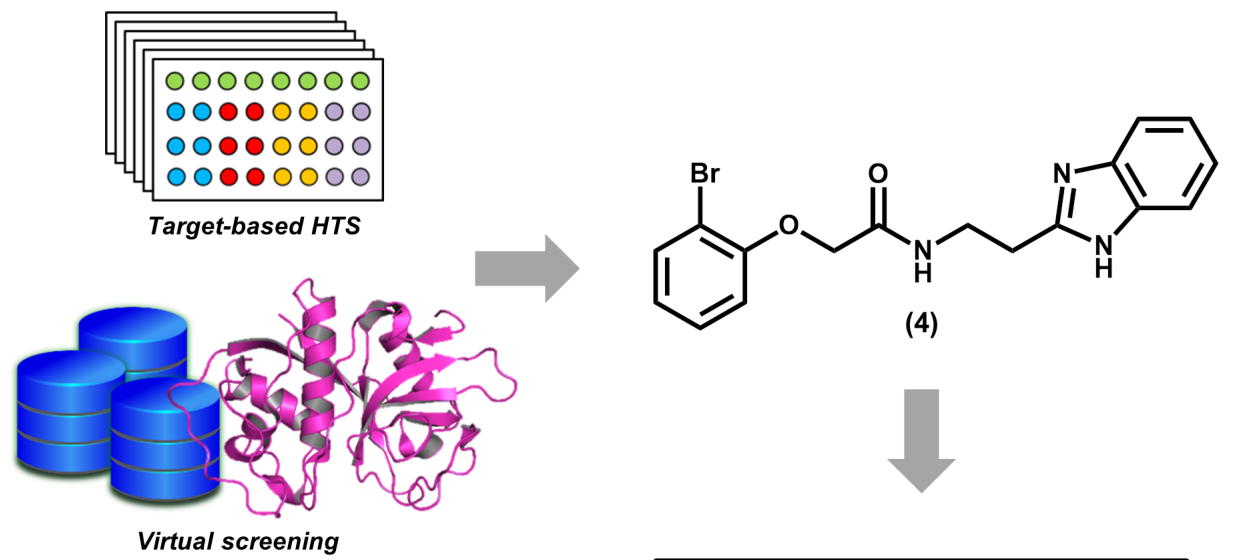

Molecular modeling Synthesis of analogs

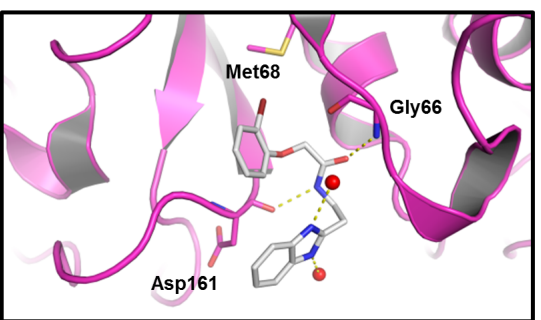

Cruzain in complex with compound 4

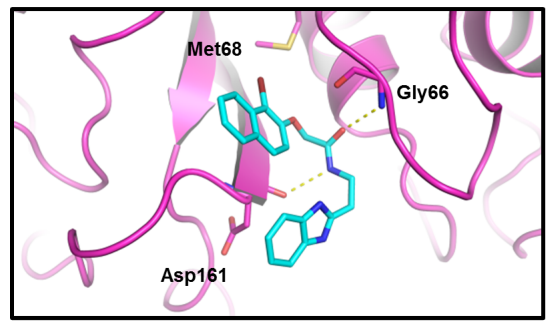<smiles>O=C(COc1ccc2ccccc2c1Br)NCCc1nc2ccccc2[nH]1</smiles>

Cruzain in complex with compound 5

Figure 3 - Integration of target-based high-throughput screening (HTS), molecular modeling and structure-activity relationship (SAR) studies for the discovery of novel reversible cruzain inhibitors.

that, given their promising biological activity and pharmacokinetics, are currently undergoing preclinical investigations aimed at evaluating their in vivo efficacy.

\section{SCHISTOSOMIASIS}

Schistosomiasis is a NTD caused by worms of the genus Schistosoma, mainly Schistosoma japonicum, Schistosoma haematobium and Schistosoma mansoni. The disease affects approximately 260 million people in nearly 80 countries, causing approximately 200,000 deaths each year. Additionally, 800 million people, mainly school-aged children, are susceptible to acquiring the infection (Colley et al. 2014). Schistosomiasis chemotherapy is based exclusively on praziquantel (PZQ), which is an old pharmacological agent identified in the 1970s (Thétiot-Laurent et al. 2013). PZQ has an acceptable safety profile and is effective toward adult parasites. However, it is inactive against immature worms, and it is quickly metabolized. Clinically relevant drug resistance has not been detected; however, a reduced susceptibility in field isolates of $S$. mansoni has been observed in distinct sites (Wang et al. 2012). These shortcomings have become serious concerns 


\section{A}
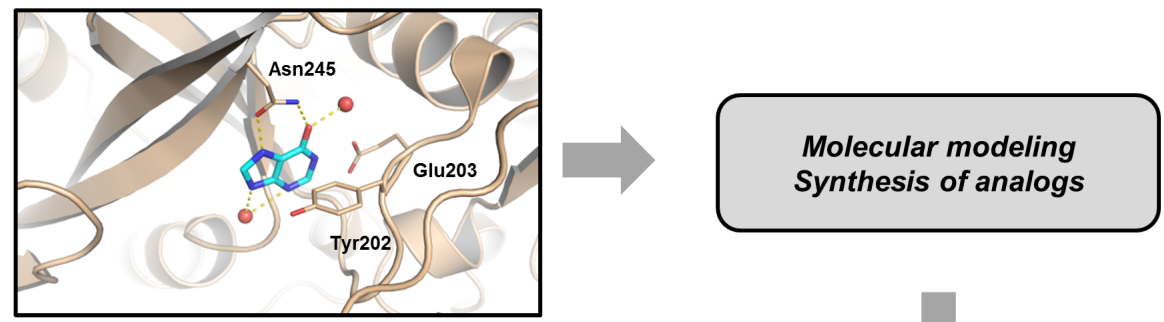

PNP in complex with hypoxanthine<smiles>CCC(C)c1ccc(Cc2c[nH]c3c(=O)[nH]c(N)nc23)cc1</smiles>

PNP in complex with compound 6

B

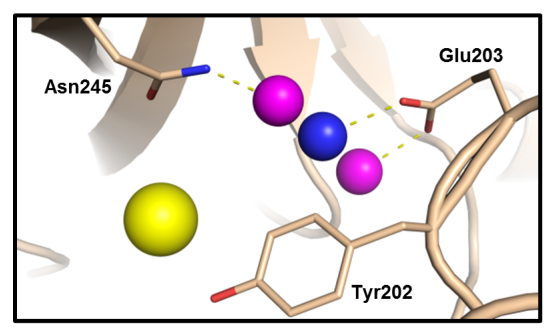

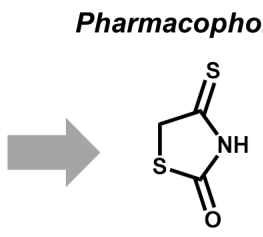

(8)<smiles>O=C1NC(=S)/C(=C/c2ccccc2O)S1</smiles>

(9)

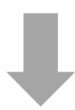<smiles>O=C1NC(=S)/C(=C/c2cccc(Br)c2)S1</smiles><smiles>O=C1NC(=S)/C(=C/c2ccc(O)cc2)S1</smiles>

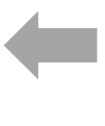

Drug design Synthesis of analogs

Figure 4 - Identification of Schistosoma mansoni purine nucleoside phosphorylase (PNP) inhibitors. (A) $\mathrm{X}$-ray crystallography and molecular docking approaches to the discovery of purine analogs as highly potent S. mansoni PNP inhibitors. (B) Pharmacophore-based virtual screening used in the identification of thioxothiazolidinone derivatives as novel $S$. mansoni PNP inhibitors.

for the scientific community and demonstrate the need for novel drugs for schistosomiasis.

Therefore, several molecular targets from the Schistosoma species have been investigated (Mafud et al. 2016). These studies have been fostered by the publication of the genome of the three main species implicated in the human disease (Berriman et al. 2009, Zhou et al. 2009, Young et al. 2012). The disclosure of these data has been a major breakthrough in the field because it has allowed for a better understanding of the molecular machinery involved in the parasite-host interplay and, consequently, in the disease physiopathology. One of the investigated targets, the enzyme purine nucleoside phosphorylase (PNP) is a key component in the purine recovery biochemical route (Pereira et al. 2010). Since Schistosoma worms lack a de novo purine biosynthesis pathway, they depend entirely on the salvage pathway to fulfill their need for purines, which are required for the biosynthesis 
of nucleic acids (Postigo et al. 2011). Following these findings, PNP has been explored at LQMC as a pharmacological target for the discovery of novel anti-schistosomal agents.

In one of these studies, potent S. mansoni PNP inhibitors were identified by an approach that combined X-ray crystallography and molecular modeling (Fig. 4a). A crystallographic structure of the enzyme previously obtained in our research group was used in molecular docking efforts by which a series of deazaguanine derivatives and other purine analogs were designed and evaluated (Castilho et al. 2010). This study led to the discovery of the highly active $S$. mansoni PNP inhibitors, 6-7, that had $\mathrm{IC}_{50}$ values of 800 and $150 \mathrm{nM}$, respectively. Some compounds from this series were found to be moderately selective for the $S$. mansoni enzyme, as assessed by counterscreening assays performed on human PNP. After enzyme inhibition experiments, an X-ray structure of compound $\mathbf{6}$ in complex with $S$. mansoni PNP was obtained. This structure enabled the observation of the key intermolecular interactions that support the activity of these inhibitors. The purine ring, for instance, was found to engage in an extensive water-mediated hydrogen bond network with Ser247 and Glu203. The pyridine ring, in turn, interacts with Tyr202. The biological data along with the structural findings gathered in this work provide useful guidelines for the design of novel $S$. mansoni PNP inhibitors.

In further studies, several crystallographic structures of $S$. mansoni PNP were used for the generation of a pharmacophore model (Fig. 4b) (Postigo et al. 2010). The pharmacophore was identified based on the available PNP-inhibitor complexes, which revealed the most usual intermolecular interactions involved in the proteinligand recognition. Next, this 3D pharmacophore was used in a virtual screening effort. Commercially available compound databases were filtered by the Lipinski's rule of five and subsequently probed using the identified pharmacophore. This virtual screening campaign led to the discovery of several thioxothiazolidinone derivatives as novel $S$. mansoni PNP inhibitors (8-9). Taking the structures of these virtual hits as references, novel analogs were designed, synthesized and evaluated. The design of this new series was based on previous SAR data that showed that 9-deazaguanine derivatives that have bulky substituents engage in favorable interactions with the binding site of PNP (Castilho et al. 2010). In short, this pharmacophorebased virtual screening led to the identification of novel $S$. mansoni PNP inhibitors that have suitable properties for further medicinal chemistry and molecular optimization studies (10-11, $K_{\mathrm{i}}$ values of 9 and $12 \mu \mathrm{M}$, respectively).

\section{QSPR MODELING AND PHARMACOKINETICS EVALUATION}

The investigation of pharmacokinetics is critical for drug discovery. A drug candidate must exhibit a suitable balance between effectiveness against the target disease and appropriate ADMET properties. However, achieving an equilibrium between so many different parameters is not a trivial task. This is corroborated by numbers showing that poor pharmacokinetics is the leading cause of attrition during the late phases of drug R\&D (Di 2015). In this scenario, it is critical to estimate the ADME profiles of drug candidates during the early stages of the discovery process. Thus, the generation of predictive QSPR models is an effective strategy that has been increasingly used in parallel with in vitro pharmacokinetics profiling (Fig. 5).

In line with our ongoing efforts focused on the establishment of integrated computational and experimental platforms to investigate drug ADME, we have developed the Database for Pharmacokinetic Properties (PK/DB), which is a tool for pharmacokinetics prediction based on highly predictive QSPR models (www.pkdb.ifsc. usp.br) (Moda et al. 2008). These models were 


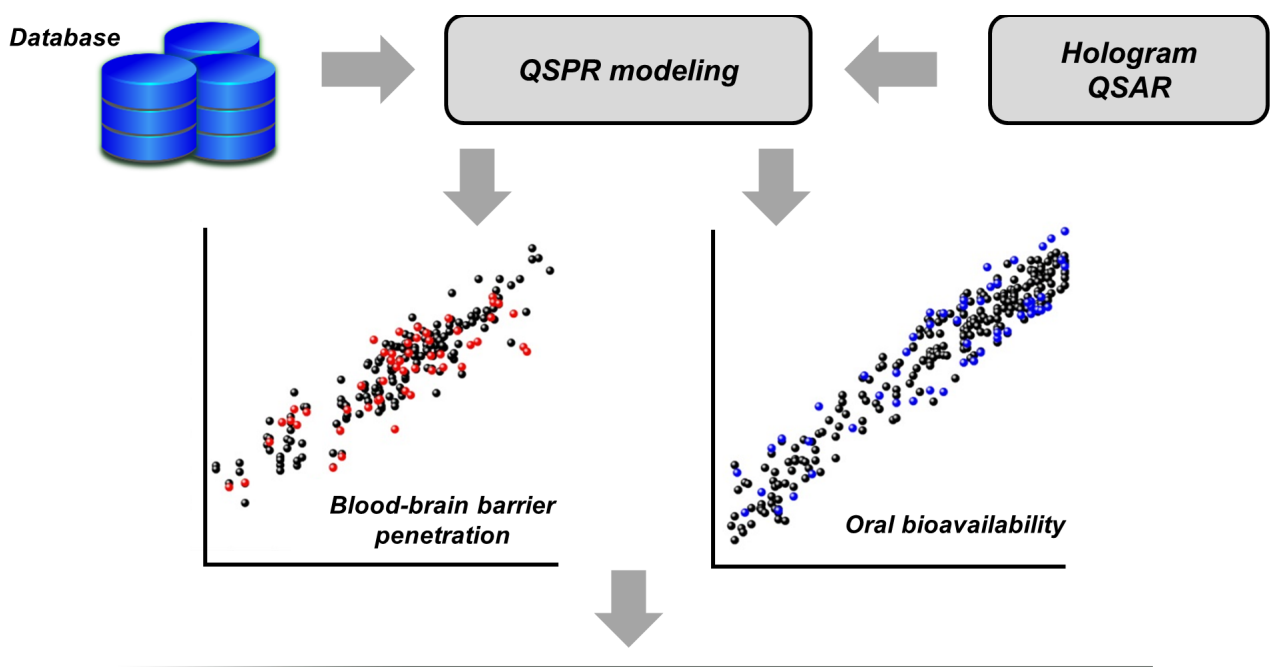

\section{$P K / D B^{\text {Database for }}$ Pharmacosinetic Properies}

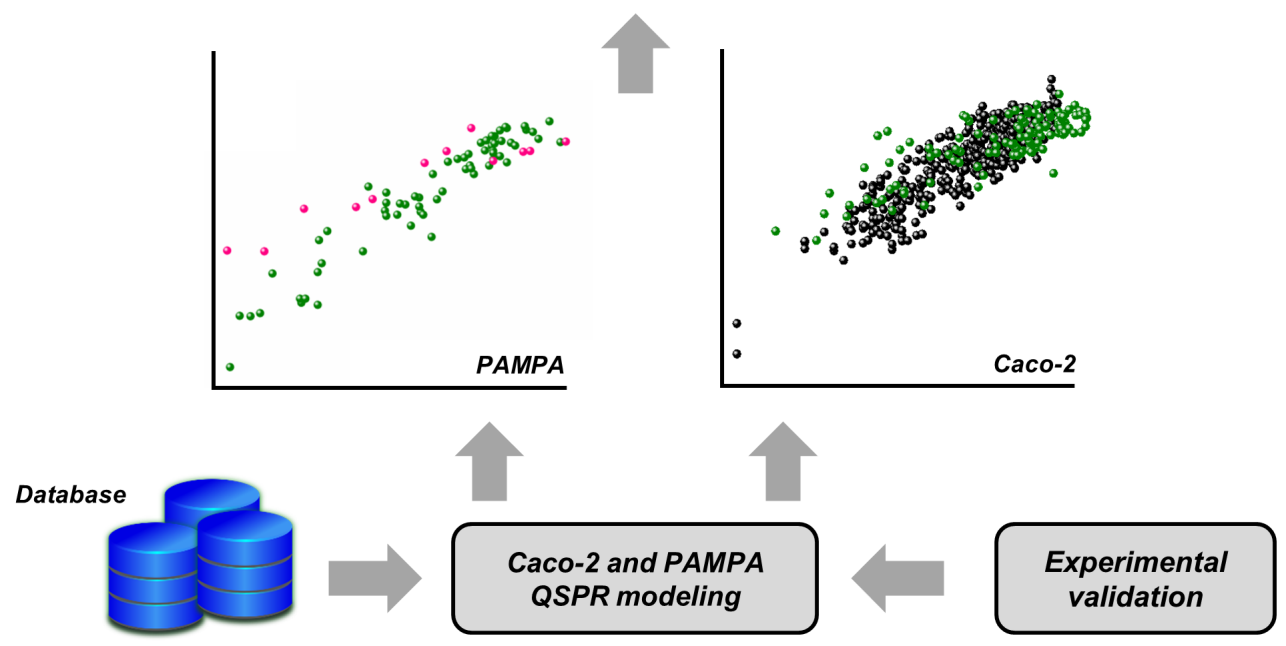

Figure 5 - Quantitative structure-property relationship (QSPR) modeling and the construction of the Database for Pharmacokinetic Properties (PK/DB).

built using the fragment-based method, Hologram QSAR (HQSAR), and using data retrieved from publicly available databases and the literature. The compilation of this material resulted in the generation of a data set that consists of more than 1200 compounds that have experimentally measured ADME properties. These data were used to construct six predictive QSPR models for the following parameters: human oral bioavailability, plasma protein binding, HIA, blood-brain barrier permeation, P-gp inhibition and water solubility.
The robustness of the resulting models was confirmed by several internal validation procedures and by the use of external test sets.

During the second stage of this project, we developed new HIA models and validated them with experimental data obtained in our laboratory. In this investigation, QSPR models to estimate the permeability in Caco-2 and PAMPA assays were generated using data from more than 500 chemically diverse compounds. Drugs covering several therapeutic classes, such as antibiotics, 
anti-hypertensives, analgesics, anti-cancer, antiviral and anti-inflammatory agents were included. Both Caco-2 and PAMPA models demonstrated a remarkable correlation ability for the training set used to construct the models, as well as a noteworthy predictive power for a test set. Moreover, the quality of these models was assessed using validation sets that consisted of cruzain inhibitors designed and evaluated for their in vitro permeability in our laboratory. Both the Caco-2 and PAMPA models exhibited significant predictive abilities for these molecules. These newly constructed QSPR models are currently being incorporated into our pharmacokinetics database in an effort towards its continual improvement. These studies illustrate the full integration between computational and experimental strategies in drug discovery, which is pivotal for the establishment of robust centers for pharmacokinetics evaluation in Brazil.

DEVELOPMENT OF A NATURAL PRODUCTS DATABASE FROM THE BIODIVERSITY OF BRAZIL

Molecules isolated from natural sources or inspired by the structure of natural products have been actively explored in drug R\&D. Recent estimates have shown that nearly $64 \%$ of all marketed drugs have their origins in natural products (Newman and Cragg 2012). In this context, the availability of well-structured and easy-to-access natural product compound collections is an important demand in current drug discovery. Therefore, the remarkably rich Brazilian biodiversity, which accounts for approximately $20 \%$ of the global biodiversity, has been consistently explored by several research groups in Brazil. Among them, the Núcleo de Bioensaios, Biossíntese e Ecofisiologia de Produtos Naturais $(\mathrm{NuBBE})$ of the Instituto de Química at the Universidade Estadual Paulista (UNESP) has been a pioneering group in the field of natural product chemistry.

In a project involving NuBBE and LQMC, we have developed a pioneering resource for drug discovery called the $\mathrm{NuBBE}$ database $\left(\mathrm{NuBBE}_{\mathrm{DB}}\right.$, freely available at http://nubbe.iq.unesp.br/ nubbeDB.html), a novel chemo-informatics tool incorporating natural products from the Brazilian biodiversity (Valli et al. 2013). Including botanical, chemical, pharmacological, and toxicological information, $\mathrm{NuBBE}_{\mathrm{DB}}$ affords accurate information to the global scientific community and is a useful resource for drug discovery studies, particularly those involving virtual screening, metabolomics, and medicinal chemistry. Given the lack of databases that include secondary metabolites from the Brazilian biodiversity, $\mathrm{NuBBE}_{\mathrm{DB}}$ meets an important need in drug discovery since the knowledge of this chemical diversity, which has been produced over many years of research, is fragmented and very challenging to access.

The information used as the input to build the database was retrieved from papers published by NuBBE, which include chemical and biological data for 640 compounds. $\mathrm{NuBBE}_{\mathrm{DB}}$ compiles a variety of information for each of these compounds, including chemical class, name, molecular formula, mass, and source. In addition, biological, pharmacological and toxicological data, and related literature are provided. Moreover, molecular descriptors, such as the number of rotatable bonds, calculated octanol/ water partition coefficients (cLogP), number of hydrogen-bond donors and acceptors, number of Lipinski's rule of five violations, topological polar surface area (TPSA), and molecular volume, are available. The compound structures are encoded as SMILES strings, and the 3D conformations are accessible as Mol2 files. Several search mechanisms are available to explore the database, including a molecular drawing interface that allows the user to filter and select molecules using a combination of properties and chemical structures. The output of a given query includes several molecular descriptors, the structure of the selected molecules, a link to download the Mol2 files, and an information table for each compound. 
One of the most innovative aspects of $\mathrm{NuBBE}_{\mathrm{DB}}$ is the inclusion of a broad diversity of information on secondary metabolites isolated from organisms from all representative Brazilian ecosystems, including the Amazon, the Atlantic Rainforest, Cerrado, and molecules from marine organisms. The chemical space covered is considerably extensive, featuring molecules from several distinct classes, such as pyrans, terpenoids, benzoic acid derivatives, alkaloids, phenylpropanoids, lignans, flavonoids, iridoids, and others. This demonstrates the high diversity and the chemical richness of $\mathrm{NuBBE}_{\mathrm{DB}}$. Approximately half of the molecules in $\mathrm{NuBBE}_{\mathrm{DB}}$ have drug-like properties, an aspect that represents an interesting profile for the identification of compounds for drug design purposes.

In short, $\mathrm{NuBBE}_{\mathrm{DB}}$ is a pioneering database that compiles information on natural products from the Brazilian biodiversity, which comprises a broad chemical diversity with an ample variety of biological and pharmacological properties. This valuable tool can be used to assist drug research programs, especially those involving chemo-informatics, virtual screening and metabolomics studies.

\section{CONCLUSIONS}

Drug development is constantly evolving as novel scientific discoveries and technological advances are incorporated into the field. More efficient organic synthesis methods, chemical biology approaches and bio- and chemoinformatics strategies have dramatically changed the process by which an initial hit is converted into a marketable drug. In fact, this endeavor has become more challenging and more complex. In part, this can be attributed to the expansion of the field towards novel therapeutic areas that are at the limits of the science and technology available today. Undeniably, the pharmaceutical industry has turned its attention to complex and multifactorial conditions, such as cancer and diseases of age. However, the technology boost resulting from these enterprises produces beneficial effects for all other areas and players involved in drug discovery. Indeed, the strategies discussed in this review are strongly dependent on the high-quality interplay between the basic research originating from research institutions and academia and the $R \& D$ expertise coming from industry. An example is our partnerships with pharmaceutical companies and not-for-profit organizations to develop novel drug candidates for Chagas disease and leishmaniasis. The establishment of this type of joint effort is of pivotal importance for the progress of drug discovery. This applies especially to therapeutic areas, such as NTDs, that still require surmounting important challenges, such as decades of lack of novelty and shortage of resources.

In this context, multidisciplinary and integrated approaches are indispensable. Research facilities able to perform experimental and computational studies to evaluate pharmacodynamics and pharmacokinetics are greatly needed to identify molecules with a high potential to become drug candidates. This scenario demands a steady input from several areas of the chemical sciences, such as organic, medicinal, and biological chemistry, which stand at the frontier of the current drug R\&D model. The integration of these disciplines in well-structured and consistent research projects is critical for developing promising drug candidates for treating critical conditions such as cancer and NTDs. Moreover, such an approach allows for considerable progress in biopharmaceutical innovation, which is a field that is a major scientific and technological bottleneck in Brazil.

\section{ACKNOWLEDGMENTS}

We gratefully acknowledge financial support from the Fundação de Amparo à Pesquisa do Estado de São Paulo (FAPESP), grants 2013/07600-3 
and 2013/25658-9, and the Conselho Nacional de Pesquisa e Desenvolvimento (CNPq), Brazil.

\section{REFERENCES}

ASHA S AND VIDYAVATHI M. 2010. Role of human liver microsomes in in vitro metabolism of drugs-a review. Appl Biochem Biotechnol 160: 1699-1722.

BAIG MH, AHMAD K, ROY S, ASHRAF JM, ADIL M, SIDDIQUI MH, KHAN S, KAMAL MA, PROVAZNÍK I AND CHOI I. 2016. Computer Aided Drug Design: Success and Limitations. Curr Pharm Des 22: 572-581.

BARANCZEWSKI P, STAŃCZAK A, SUNDBERG K, SVENSSON R, WALLIN A, JANSSON J, GARBERG $P$ AND POSTLIND H. 2006. Introduction to in vitro estimation of metabolic stability and drug interactions of new chemical entities in drug discovery and development. Pharmacol Rep 58: 453-472.

BERGSTRÖM CA ET AL. 2014. Early pharmaceutical profiling to predict oral drug absorption: current status and unmet needs. Eur J Pharm Sci 57: 173-199.

BERHANU WM, PILLAI GG, OLIFERENKO AA AND KATRITZKY AR. 2012. Quantitative structure-activity/ property relationships: the ubiquitous links between cause and effect. ChemPlusChem 77: 507-517.

BERRIMAN M ET AL. 2009. The genome of the blood fluke Schistosoma mansoni. Nature 460: 352-358.

CASTILHO MS, GUIDO RVC AND ANDRICOPULO AD. 2009. 2D Quantitative structure-activity relationship studies on a series of cholesteryl ester transfer protein inhibitors. Bioorg Med Chem 15: 6242-6252.

CASTILHO MS, POSTIGO MP, PEREIRA HM, OLIVA G AND ANDRICOPULO AD. 2010. Structural basis for selective inhibition of purine nucleoside phosphorylase from Schistosoma mansoni: Kinetic and structural studies. Bioorg Med Chem 18: 1421-1427.

COLLEY DG, BUSTINDUY AL, SECOR WE AND KING CH. 2014. Human schistosomiasis. Lancet 383: 2253-2264.

COPELAND RA. 2000. Enzymes: a practical introduction to structure, mechanism, and data analysis, New York: Wiley VHC, $104 \mathrm{p}$.

COURA JR AND BORGES-PEREIRA J. 2011. Chronic phase of Chagas disease: why should it be treated? A comprehensive review. Mem Inst Oswaldo Cruz 106: 641645.

DI L. 2015. Strategic approaches to optimizing peptide ADME properties. AAPS J 17: 134-143.

DOPAZO J. Genomics and transcriptomics in drug discovery. 2014. Drug Discov Today 19: 126-132.

DOYLE PS, ZHOU YM, ENGEL JC AND MCKERROW JH. 2007. A cysteine protease inhibitor cures Chagas' disease in an immunodeficient-mouse model of infection. Antimicrob Agents Chemother 51: 3932-3939.
DOYLE PS, ZHOU YM, HSIEH I, GREENBAUM DC, MCKERROW JH AND ENGEL JC. 2011. The Trypanosoma cruzi protease cruzain mediates immune evasion. PLoS Pathog 7: e1002139.

EDER J, SEDRANI R AND WIESMANN C. 2014. The discovery of first-in-class drugs: origins and evolution. Nat Rev Drug Discov 13: 577-587.

FERREIRA LG AND ANDRICOPULO AD. 2016. Drug repositioning approaches to parasitic diseases: a medicinal chemistry perspective. Drug Discov Today 21: 1699-1710.

FERREIRA LG AND ANDRICOPULO AD. 2017. Targeting cysteine proteases in trypanosomatid disease drug discovery. Pharmacol Ther S0163-7258: 30152-3.

FERREIRA LG, DE OLIVEIRA MT AND ANDRICOPULO AD. 2016. Advances and Progress in Chagas Disease Drug Discovery. Curr Top Med Chem 16: 2290-2302.

FERREIRA RS, DESSOY MA, PAULI I, SOUZA ML, KROGH R, SALES AI, OLIVA G, DIAS LC AND ANDRICOPULO AD. 2014. Synthesis, biological evaluation, and structure-activity relationships of potent noncovalent and nonpeptidic cruzain inhibitors as antiTrypanosoma cruzi agents. J Med Chem 57: 2380-2392.

FERREIRA LG, DOS SANTOS RN, OLIVA G AND ANDRICOPULO AD. 2015. Molecular docking and structure-based drug design strategies. Molecules 20: 13384-13421.

FERREIRA RS ET AL. 2010. Complementarity between a docking and a high-throughput screen in discovering new cruzain inhibitors. J Med Chem 53: 4891-4905.

FOUAD YA AND AANEI C. 2017. Revisiting the hallmarks of cancer. Am J Cancer Res 7: 1016-1036.

GALETIN A, GERTZ M AND HOUSTON JB. 2010. Contribution of intestinal cytochrome p450-mediated metabolism to drug-drug inhibition and induction interactions. Drug Metab Pharmacokinet 25: 28-47.

GLOBAL BURDEN OF DISEASE CANCER COLLABORATION ET AL. 2015. The Global Burden of Cancer 2013. JAMA Oncol 1: 505-527.

HONÓRIO KM, MODA TL AND ANDRICOPULO AD. 2013. Pharmacokinetic properties and in silico ADME modeling in drug discovery. Med Chem 9: 163-176.

HOWAT S, PARK B, OH IS, JIN YW, LEE EK AND LOAKE GJ. 2014. Paclitaxel: biosynthesis, production and future prospects. N Biotechnol 31: 242-245.

JULIANO RL. 2013. Pharmaceutical innovation and public policy: The case for a new strategy for drug discovery and development. Sci Publ Policy 40: 393-405.

KAMAL A, ALI HUSSAINI SM, RAHIM A AND RIYAZ S. 2015. Podophyllotoxin derivatives: a patent review (2012 - 2014). Expert Opin Ther Pat 25: 1025-1034.

KIRCHMAIR J, GÖLLER AH, LANG D, KUNZE J, TESTA B, WILSON ID, GLEN RC AND SCHNEIDER G. 
2015. Predicting drug metabolism: experiment and/or computation? Nat Rev Drug Discov 14: 387-404.

LECLAND N AND LÜDERS J. 2014. The dynamics of microtubule minus ends in the human mitotic spindle. Nat Cell Biol 16: 770-778.

MAFUD AC, FERREIRA LG, MASCARENHAS YP, ANDRICOPULO AD AND DE MORAES J. 2016. Discovery of Novel Antischistosomal Agents by Molecular Modeling Approaches. Trends Parasitol 32: 874-886.

MAGALHAES LG, MARQUES FB, DA FONSECA MB, ROGÉRIO KR, GRAEBIN CS AND ANDRICOPULO AD. 2016. Discovery of a Series of Acridinones as Mechanism-Based Tubulin Assembly Inhibitors with Anticancer Activity. PLoS One 11: e0160842.

MANNE-GOEHLER J, UMEH CA, MONTGOMERY SP AND WIRTZ VJ. 2016. Estimating the Burden of Chagas Disease in the United States. PLoS Negl Trop Dis 10: e0005033.

MEDANI S AND WALL C. 2016. Colchicine toxicity in renal patients - Are we paying attention? Clin Nephrol 86: 100105.

MODA TL, TORRES LG, CARRARA AE AND ANDRICOPULO AD. 2008. PK/DB: database for pharmacokinetic properties and predictive in silico ADME models. Bioinformatics 24: 2270-2271.

MODA TL AND ANDRICOPULO AD. 2012. Consensus hologram QSAR modeling for the prediction of human intestinal absorption. Bioorg Med Chem Lett 22: 28892893.

MUKHTAR E, ADHAMI VM AND MUKHTAR H. 2014. Targeting microtubules by natural agents for cancer therapy. Mol Cancer Ther 13: 275-284.

NEWMAN DJ AND CRAGG GM. 2012. Natural products as sources of new drugs over the 30 years from 1981 to 2010. Nat Prod 75: 311-335.

OVERTON TW. 2014. Recombinant protein production in bacterial hosts. Drug Discov Today 19: 590-601.

PAULI I, FERREIRA LG, DE SOUZA ML, OLIVA G, FERREIRA RS, DESSOY MA, SLAFER BW, DIAS LC AND ANDRICOPULO AD. 2017. Molecular modeling and structure-activity relationships for a series of benzimidazole derivatives as cruzain inhibitors. Future Med Chem 9: 641-657.

PEREIRA HM, BERDINI V, FERRI MR, CLEASBY A AND GARRATT RC. 2010. Crystal structure of Schistosoma purine nucleoside phosphorylase complexed with a novel monocyclic inhibitor. Acta Trop 114: 97-102.

POSTIGO MP, GUIDO RV, OLIVA G, CASTILHO MS, DA R PITTA I, DE ALBUQUERQUE JF AND ANDRICOPULO AD. 2010. Discovery of new inhibitors of Schistosoma mansoni PNP by pharmacophore-based virtual screening. J Chem Inf Model 50: 1693-1705.
POSTIGO MP, KROGH R, TERNI MF, PEREIRA HM, OLIVA G, CASTILHO MS AND ANDRICOPULO AD. 2011. Enzyme kinetics, structural analysis and molecular modeling studies on a series of Schistosoma mansoni PNP inhibitors. J Braz Chem Soc 22: 583-591.

SELIGMANN J AND TWELVES C. 2013. Tubulin: an example of targeted chemotherapy. Future Med Chem 5: 339-352.

STANTON DT. 2012. QSAR and QSPR model interpretation using partial least squares (PLS) analysis. Curr Comput Aided Drug Des 8: 107-127.

STARR A, GRAEF KM AND DENT J. 2016. Fostering innovative product development for neglected tropical diseases through partnerships. Pharm Pat Anal 5: 391-400.

SURADE S AND BLUNDELL TL. 2012. Structural biology and drug discovery of difficult targets: the limits of ligandability. Chem Biol 19: 42-50.

SWINNEY DC. 2013. Phenotypic vs. target-based drug discovery for first-in-class medicines. Clin Pharmacol Ther 93: 299-301.

SWINNEY DC AND ANTHONY J. 2011. How were new medicines discovered? Nat Rev Drug Discov 10: 507-519.

TABOUREAU O, BAELL JB, FERNÁNDEZ-RECIO J AND VILLOUTREIX BO. 2012. Established and emerging trends in computational drug discovery in the structural genomics era. Chem Biol 19: 29-41.

TESTA B, PEDRETTI A AND VISTOLI G. 2012. Reactions and enzymes in the metabolism of drugs and other xenobiotics. Drug Discov Today 17: 549-560.

THÉTIOT-LAURENT SAL, BOISSIER J, ROBERT A AND MEUNIER B. 2013. Schistosomiasis chemotherapy. Angew Chem Int Ed Engl 52: 7936-7956.

TROSSINI GHG, GUIDO RVC, OLIVA G AND ANDRICOPULO AD. 2009. Quantitative structureactivity relationships for a series of inhibitors of cruzain from Trypanosoma cruzi: Molecular modeling, CoMFA and CoMSIA studies. J Mol Graph Model 28: 3-11.

VALLI M, DOS SANTOS RN, FIGUEIRA LD, NAKAJIMA CH, CASTRO-GAMBOA I, ANDRICOPULO AD AND BOLZANI VS. 2013. Development of a natural products database from the biodiversity of Brazil. J Nat Prod 76: 439-444.

VOLPE DA. 2011. Drug-permeability and transporter assays in Caco-2 and MDCK cell lines. Future Med Chem 3: 2063-2077.

WANG W, WANG L AND LIANG YS. 2012. Susceptibility or resistance of praziquantel in human schistosomiasis: a review. Parasitol Res 111: 1871-1877.

YOUNG ND ET AL. 2012. Whole-genome sequence of Schistosoma haematobium. Nat Genet 44: 221-225.

ZHOU Y ET AL. 2009. The Schistosoma japonicum genome reveals features of host-parasite interplay. Nature 460: 345-351. 